\title{
Crystal structure of bis(aniline)dichlorocobalt, $\left[\left(\mathrm{C}_{6} \mathrm{H}_{5} \mathrm{NH}_{2}\right)_{2} \mathrm{CoCl}_{2}\right]$
}

\author{
R. A. Burrow* \\ University of Toronto. Deparment of Chemistry. Toronto. Ontario. Canada M5S 3H6.
}

\section{Hörner, L. Schultz Lang}

Universidade Federal de Santa Maria. Departamento de Química, Santa Maria, Rio Grande do Sul, Brasil. 97119-900

\section{A. Neves and I. Vencato}

Universidade Federal de Santa Catarina. Departamento de Química, Forinópolis, Santa Catarina, Brasil, 88010-970

Received May 29, 1996, CSD-No. 402496

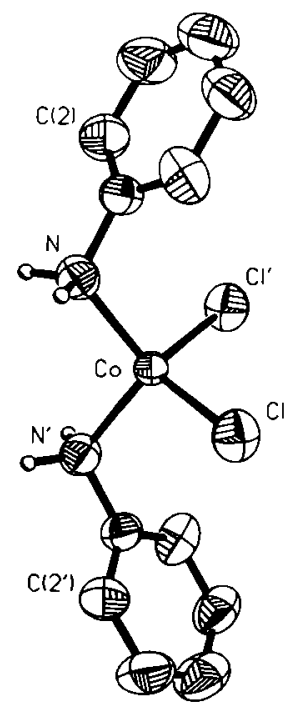

Source of material: The compound is prepared by the reaction of disodium 1,3-bis(phenyltriazenide)benzene (see ref. 1) with $\mathrm{CoCl}_{2} \cdot 6 \mathrm{H}_{2} \mathrm{O}$ in tetrahydrofuran upon addition of ethanol and standing in air several days.

The molecule has the symmetry 2 . The aniline and chloride ligands form a near tetrahedral arrangement about the Co center as predicted from powder X-ray data (see ref. 2) and is similar to the structure of the related complex dichloro-di( $p$-toluidine)cobalt (see refs. 3, 4).
$\mathrm{C}_{12} \mathrm{H}_{14} \mathrm{Cl}_{2} \mathrm{CoN}_{2}$, monoclinic, $C 12 / c 1$ (No. 15), $a=26.142(6) \AA$, $b=4.801(1) \AA, c=11.607(2) \AA, \beta=108.82(3)^{\circ}, V=1378.9 \AA^{3}, Z=4$, $R(F)=0.061, R_{W}(F)=0.136$.

Table 1. Parameters used for the X-ray data collection

\begin{tabular}{ll} 
Crystal: & red-brown plate, size $0.05 \times 0.15 \times 0.30 \mathrm{~mm}$ \\
Wavelength: & Mo $K_{\alpha}$ radiation $(0.71073 \AA)$ \\
$\mu:$ & $16.11 \mathrm{~cm}^{-1}$ \\
Diffractometer: & Enraf-Nonius CAD4 \\
Scan mode: & $\omega / 2 \theta$ \\
$T_{\text {measuremen: }}$ & $293 \mathrm{~K}$ \\
$2 \theta_{\text {max }}$ & $50^{\circ}$ \\
N(hkl) & 1205 \\
Criterion for $I_{0}:$ & $I_{0}>2 \sigma\left(I_{0}\right)$ \\
N(param)refined: & 88 \\
Program: & SHELXTL-plus \\
\hline
\end{tabular}

Table 2. Final atomic coordinates and displacement parameters (in $\AA^{2}$ )

\begin{tabular}{llllll}
\hline Atom & Site & $x$ & $y$ & $z$ & Uiso \\
\hline Hn(A) & $8 f$ & $-0.061(3)$ & $-0.36(2)$ & $0.248(7)$ & $0.05(3)$ \\
$\mathrm{Hn}(\mathrm{B})$ & $8 f$ & $-0.048(4)$ & $-0.24(2)$ & $0.376(8)$ & $0.07(3)$ \\
$\mathrm{H}(2 \mathrm{~A})$ & $8 f$ & $-0.1434(4)$ & $-0.233(2)$ & $0.0982(9)$ & $0.13(2)$ \\
$\mathrm{H}(3 \mathrm{~A})$ & $8 f$ & $-0.2246(5)$ & $0.006(3)$ & $0.049(1)$ & $0.13(2)$ \\
$\mathrm{H}(4 \mathrm{~A})$ & $8 f$ & $-0.2394(5)$ & $0.327(3)$ & $0.187(1)$ & $0.13(2)$ \\
H(5A) & $8 f$ & $-0.1715(5)$ & $0.431(3)$ & $0.362(1)$ & $0.13(2)$ \\
H(6A) & $8 f$ & $-0.0898(5)$ & $0.195(2)$ & $0.4125(9)$ & $0.13(2)$ \\
\hline
\end{tabular}

Table 3. Final atomic coordinates and displacement parameters (in $\AA^{2}$ )

\begin{tabular}{|c|c|c|c|c|c|c|c|c|c|c|}
\hline Atom & Site & $x$ & $y$ & $z$ & $U_{11}$ & $U_{22}$ & $U_{33}$ & $U_{12}$ & $U_{13}$ & $U_{\mathbf{2 3}}$ \\
\hline Co & $4 e$ & 0 & $0.0555(4)$ & $1 / 4$ & $0.031(1)$ & $0.0336(9)$ & $0.0345(9)$ & 0 & $0.0123(7)$ & $\mathbf{0}$ \\
\hline $\mathrm{Cl}$ & $8 f$ & $0.0415(1)$ & $0.3261(5)$ & $0.4108(2)$ & $0.068(2)$ & $0.056(2)$ & $0.047(1)$ & $-0.000(1)$ & $0.016(1)$ & $-0.004(1)$ \\
\hline $\mathbf{N}$ & $8 f$ & $-0.0571(3)$ & $-0.184(2)$ & $0.2896(8)$ & $0.051(6)$ & $0.046(5)$ & $0.053(5)$ & $0.003(4)$ & $0.017(4)$ & $-0.002(4)$ \\
\hline$C(1)$ & $8 f$ & $-0.1087(4)$ & $-0.045(2)$ & $0.2610(8)$ & $0.040(6)$ & $0.049(5)$ & $0.064(6)$ & $0.003(5)$ & $0.017(5)$ & $0.010(5)$ \\
\hline$C(2)$ & $8 f$ & $-0.1490(4)$ & $-0.101(2)$ & $0.1516(9)$ & $0.054(7)$ & $0.066(8)$ & $0.061(6)$ & $-0.001(6)$ & $0.002(5)$ & $-0.001(6)$ \\
\hline$C(3)$ & $8 f$ & $-0.1976(5)$ & $0.041(3)$ & $0.123(1)$ & $0.053(8)$ & $0.10(1)$ & $0.090(8)$ & $0.007(8)$ & $0.003(6)$ & $0.014(8)$ \\
\hline
\end{tabular}


Table 3. (Continued)

\begin{tabular}{lllllllllll}
\hline Alom & Site & $x$ & $y$ & $z$ & $U_{11}$ & $U_{22}$ & $U_{33}$ & $U_{12}$ & $U_{13}$ & $U_{23}$ \\
\hline $\mathrm{C}(5)$ & $8 f$ & $-0.1661(5)$ & $0.296(3)$ & $0.310(1)$ & $0.069(9)$ & $\mathbf{0 . 0 6 9 ( 8 )}$ & $\mathbf{0 . 0 8 7 ( 8 )}$ & $\mathbf{0 . 0 2 0 ( 7 )}$ & $0.037(7)$ & $0.011(7)$ \\
$\mathrm{C}(6)$ & $8 f$ & $-0.1170(5)$ & $0.155(2)$ & $\mathbf{0 . 3 4 0 0 ( 9 )}$ & $0.080(8)$ & $\mathbf{0 . 0 6 3 ( 7 )}$ & $\mathbf{0 . 0 5 3 ( 6 )}$ & $\mathbf{0 . 0 1 7 ( 6 )}$ & $\mathbf{0 . 0 3 7 ( 6 )}$ & $0.013(5)$ \\
\hline
\end{tabular}

Acknowledgments. Financial assistance provided by FAPERGS, CAPES and CNPq (Brasil), and Volkswagen-Stifung and GTZ (Germany) is greatly appreciated. We thank Dr A. Lough (Toronto) for aid in obtaining literature.

\section{References}

1. Hörner, M.; Fenner, H.; Beck, J.; Hiller, W.: Darstellung und Kristallstruktur von Tris[1.3-bis(phenyltriazenide)benzol]dithallium(III). Z. Anorg. Allg. Chem. 571 (1989) 69-74.
2. Maslennikova, I. S.: Smimova, T. N.: The structures of cobalt and nickel chloride complexes with aniline. Zh. Fiz. Khim. 52 (1978) 1788-1789.

3. Malinkovskii, T. I.: X-ray investigation of bis(p-toliduine)cobalt dichloride. Kristallografiya 2 (1957) 734-741.

4. Malinkovskii, T. I.: Structure of crystals of the type $M X_{2} \cdot 2 A$. Nauchn. Sessiya Akad. Mold. SSR, Kishinev, Sb. (1962) 135-144.

5. Sheldrick, G. M.: SHELXTL/PC V5.0. Siemens Analytical X-ray Instruments Inc., Madison, Wisconsin, U.S.A. 1995.

6. Enraf-Nonius: CAD4 Express Software. Enraf-Nonius Inc., Delft, The Netherlands 1992. 